\title{
BASIC SCIENCE ARTICLE Effect of polyunsaturated fatty acids on postnatal ileum development using the fat-1 transgenic mouse model
}

\author{
Pratibha Singh ${ }^{1}$, Pedro Ochoa-Allemant ${ }^{1}$, Joanne Brown ${ }^{1}$, George Perides ${ }^{1}$, Steven D. Freedman ${ }^{1}$ and Camilia R. Martin $^{2}$
}

\begin{abstract}
BACKGROUND: Long-chain polyunsaturated fatty acids (LCPUFAs) play a critical role in neonatal health. We hypothesized that LCPUFAs play an essential role in priming postnatal gut development. We studied the effect of LCPUFAs on postnatal gut development using fat-1 transgenic mice, which are capable of converting n-6 to n-3 LCPUFAs, and wild-type (WT) C57BL/6 mice. METHODS: Distal ileum sections were collected from fat-1 and WT mice on days 3, 14, and 28. Fatty acid analyses, histology, RT-qPCR and intestinal permeability were performed.

RESULTS: Fat-1 mice, relative to WT mice, showed increased n-3 LCPUFAs levels (a-linolenic acid, docosahexaenoic acid, and eicosapentaenoic acid, $p<0.05)$ and decreased arachidonic acid levels $(p<0.05)$ in the ileum. Preweaning fat- 1 mice, compared to WT, showed $>50 \%$ reduced muc2, Tff3, TLR9, and Camp expression $(p<0.05)$, markers of the innate immune response. There was a $>$ two-fold increased expression of Fzd5 and EphB2, markers of cell differentiation $(p<0.05)$, and Fabp2 and 6, regulators of fatty acid transport and metabolism $(p<0.05)$. Despite reduced expression of tight junction genes, intestinal permeability in fat- 1 was comparable to WT mice.
\end{abstract}

CONCLUSIONS: Our data support the hypothesis that fatty acid profiles early in development modulate intestinal gene expression in formative domains, such as cell differentiation, tight junctions, other innate host defenses, and lipid metabolism.

Pediatric Research (2019) 85:556-565; https://doi.org/10.1038/s41390-019-0284-0

\section{INTRODUCTION}

Compared to term infants, preterm infants are at an increased risk of gastrointestinal complications including feeding intolerance, necrotizing enterocolitis (NEC), and intestinal bacterial translocation leading to sepsis. ${ }^{1,2}$ Altered postnatal gut development, innate host defense, and intestinal barrier are thought to, in part, explain the increased risk of gastrointestinal morbidities in preterm infants. ${ }^{3,4}$ Gut development and its physiology are highly adaptive to nutritional changes and early intestinal adaptation may persist later in life and affect the responsiveness of the gut to physiological and pathological challenges.

Long-chain polyunsaturated fatty acids (LCPUFAs) are critical nutrients for optimal fetal development. However, in the preterm infant the exposure to these nutrients is abruptly terminated and the current nutritional practices in the neonatal intensive care unit (NICU) fail to maintain in utero levels leading to a deficit in LCPUFAs, notably docosahexaenoic acid (DHA) and arachidonic acid (AA). These deficits are linked to common neonatal morbidities, including bronchopulmonary dysplasia, nosocomial sepsis, and retinopathy of prematurity., ${ }^{5,6}$ LCPUFAs have been shown to play an immunomodulatory role in diseases of gastrointestinal origin such as inflammatory bowel disease. ${ }^{7}$ However, the role of fatty acids on the intestinal development of a preterm newborn is not well understood. The immunomodulatory function of LCPUFAs has been demonstrated in vitro using human fetal intestinal epithelial cells, as a model for the human fetal intestine. Wijendran et al. have shown that, in human fetal and adult intestinal epithelial cells, DHA supplementation results in an attenuated interleukin- $1 \beta$-induced pro-inflammatory response confirming its anti-inflammatory effect. This effect was more pronounced in fetal cells supporting its potential role in modulating intestinal inflammation, which is central to the pathogenesis of NEC. ${ }^{8}$ Animal studies have shown that supplementation of LCPUFAs decreased the incidence of NEC by downregulating the expression of Toll-like receptor 4 (TLR4), platelet-activating factor, leukotriene B4, and nuclear factor-KB signaling pathways. ${ }^{9,10}$ However, their contribution to specific postnatal intestinal developmental processes before an inciting event remains unexplored. Understanding the priming effect of enteral fatty acids on intestinal development during a preinjurious state will offer mechanisms by which fatty acids may modulate the risk of intestinal injury.

The challenge in examining the effect of a specific nutrient on host responses is that diets are composed of a complex matrix and any differences in dietary composition can lead to confounding and unclear interpretations of the data. We took advantage of a transgenic mouse model in which the fat-1 gene from Caenorhabditis elegans was inserted into the mouse genome. The fat-1 transgenic mice are capable of converting $n-6$ fatty acids to $n-3$ fatty acids and exhibit a lower ratio of $n-6: n-3$ in various tissues regardless of their diet. ${ }^{11}$

We hypothesized that LCPUFAs play an essential role in priming postnatal gut development by modulating the expression levels of various gut development markers involved in the intestinal barrier, cellular differentiation, and innate host defenses such as antimicrobial peptides. Our objective was to investigate the morphological and molecular differences in postnatal intestinal development in response to changing LCPUFA profiles that reflect the current

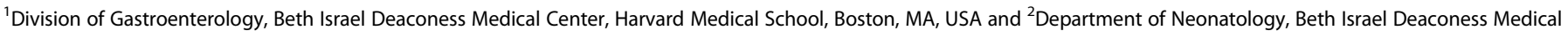
Center, Harvard Medical School, Boston, MA, USA

Correspondence: Camilia R. Martin (cmartin1@bidmc.harvard.edu)

Received: 9 August 2018 Revised: 5 December 2018 Accepted: 18 December 2018

Published online: 17 January 2019 
experience of the preterm infant in the NICU. To meet this objective, we used the fat-1 transgenic mouse model and examined intestinal morphological growth and changes in gene expression across different postnatal ages (days 3,14, and 28) compared to wild-type (WT) control mice. We chose to focus on the ileum as this is a primary site of injury in the neonatal gut. Our results begin to elucidate the role of LCPUFAs in gut morphology and in the transcriptional regulation of several genes involved in gut development.

\section{METHODS}

Animal rearing, model selection, nutrition, and sample collection All animal studies were approved by the Institutional Animal Care and Use Committee at Beth Israel Deaconess Medical Center. C57BL/6 WT mice were purchased from Jackson Laboratories (Bar Harbor, ME). Transgenic fat- 1 mice on a C57BI/ 6 background were kindly provided by Dr. Jing Kang, Massachusetts General Hospital, Boston, MA. The animals were kept under standard laboratory conditions on a controlled 12-h dark-light cycle, with unlimited access to food and water.

At approximately 2 weeks, the mouse intestinal development is equivalent to that of a 24-week gestational age infant. The mouse intestinal development reaches that of a full-term infant at approximately 4 weeks of age. ${ }^{12}$ We chose to study three time points-day 3 and 14-to reflect the intestinal stage of the most immature infants who are at greatest risk of neonatal morbidities and day 28 to reflect the full-term infant.

WT and fat-1 mothers were maintained on an enriched saturated and monounsaturated fatty acid diet (AIN-76A with $10 \%$ corn oil) from TestDiet (St. Louis, MO) before, during, and after pregnancy. The detailed composition of this diet is shown in Supplemental Table S1. WT and fat-1 pups were dam fed till weaning. Fatty acid analysis of the dam milk is summarized by genotype in Supplemental Table S2. After weaning, the WT and fat-1 mice were fed the same enriched diet used for their mothers until the end of the experiment. This diet is also known as a "western diet" and in a WT mouse the diet induces an $n-6$ dominant fatty acid profile and, in contrast, induces a n-3 dominant fatty acid profile in a fat-1 mouse due to its unique metabolism. The differences in the genotype specific fatty acid profiles allows for comparative analyses on the impact of fatty acids and the n-3:n-6 ratio on ileum development.

Mice at different ages (3-, 14-, and 28-day old) were euthanized by $\mathrm{CO}_{2}$ asphyxiation and samples collected. The first $2 \mathrm{~cm}$ of ileum proximal to the cecum was used for quantitative reverse transcription polymerase chain reaction (RT-qPCR) or histology studies. The second $2 \mathrm{~cm}$ ileal section was used for fatty acid profile analysis. The tissue samples collected were stored at $-80^{\circ} \mathrm{C}$. The samples obtained for histology were fixed in $10 \%$ formalin embedded in paraffin and $5 \mu \mathrm{m}$ sections were subjected to staining as described below.

\section{Genotyping and phenotyping}

The genotype of the mouse pups was confirmed by PCR and validated by the phenotypic change in fatty acid profiles from tail samples. Genotyping was performed using mouse tail DNA. For DNA isolation, the mouse tail was incubated with $100 \mu \mathrm{l}$ of $50 \mathrm{mM}$ $\mathrm{NaOH}$ at $95^{\circ} \mathrm{C}$. After $1 \mathrm{~h}, 10 \mu \mathrm{l}$ of $1 \mathrm{M}$ Tris- $\mathrm{HCl}(\mathrm{pH} 8.0)$ was added. The samples were vortexed, centrifuged for $15 \mathrm{~min}$ at $14,000 \times g$, at $4{ }^{\circ} \mathrm{C}$. The following primers were used: fat-1 forward, TGTTCATGCCTTCTTCTIITCC; fat-1 reverse, GCGACCATACCTCA AACTTGGA, and GoTaq ${ }^{\circledR}$ Green Master Mix (Promega, Madison, WI). PCR products and DNA ladder were subjected to gel electrophoresis on a $2 \%$ agarose gel. Our PCR results showed the presence of the fat- 1 gene only in fat-1 transgenic mice, while WT mice do not possess the fat-1 gene (Supplemental Figure S1). The assigned genotypes were confirmed by examining the induced fatty acid profiles in the tail (Supplemental Table S3).
Pups who matched the genotype of their mothers were selected for final analysis (e.g., WT pups born to WT mothers, and fat-1 pups born to fat- 1 mothers) to maintain the gene-induced fatty acid phenotypes throughout the preweaning period.

Dam milk collection

Mouse milk was collected using a homemade suction apparatus consisting of two tubing segments and a $50 \mathrm{ml}$ conical tube. Suction was created using the first tubing segment where one end was connected to vacuum at negative pressures between -18 and $-20 \mathrm{~mm} \mathrm{Hg}$ and the other end of the tubing was inserted into a sealed $50 \mathrm{~mL}$ conical tube. The second tubing segment was used as the collection system. One end was inserted into the sealed $50 \mathrm{~mL}$ conical tube and the hub end of the tubing was placed around the teat creating a seal during the milking process.

The milking process of the dams was carried out at 10-14 days' post-parturition. The dams were separated from their pups for $4-5 \mathrm{~h}$ before milking. Isoflurane (Zoetis, Parsippany, NJ) was used for anesthesia. Dams were given 5 IU of oxytocin (Sigma-Aldrich, St. Louis, MO) intraperitoneally to induce milk flow. After 3-5 min, gentle finger massage around the mammary glands was used to stimulate milk ejection. The milk was stored at $-80^{\circ} \mathrm{C}$ until further analysis.

Determination of fatty acid profiles in tail and ileum

Tissue fatty acids in the tail and distal ileum were isolated and methylated using a modified Folch method as described previously. ${ }^{13}$ Total lipids were extracted and quantified by gas chromatography-mass spectroscopy. Peak identification was based upon a comparison of both retention time and mass spectra of the unknown peak to that of known standards. Fatty acid methylated ester (FAME) mass was determined by comparing areas of unknown FAMEs to that of a fixed concentration of 17:0 internal standard. Individual fatty acids are expressed as a percentage of the total fatty acid mass (mol\%). The ratio of all $n-3$ fatty acids to all $n-6$ fatty acids was calculated and expressed as an unitless number.

\section{Intestinal morphometry}

Paraffin-embedded sections were stained with hematoxylin and eosin (H\&E) staining. The height of intact villi and depth of crypts were measured using the Aperio ImageScope software (Leica Biosystems, Buffalo Grove, IL). In every section, at least five randomly selected areas were counted to minimize sectioning variance. Villous height was measured in continuously intact villous that ended with a rounded tip at $\times 20$ magnification from the tip of villous to the entrance of the crypt opening. The depth of 20 crypts per mouse was measured for every section. Crypt depth was measured from crypt base to the villous-crypt junction.

Distal ileum sections were stained with Alcian Blue $(A B)$ and periodic acid-Schiff (PAS, Sigma-Aldrich) to stain acidic and neutral mucins. Neutral mucins stained magenta and acidic mucins stained blue. AB- and PAS-positive goblet cells were counted per 100 villous epithelial cells. Villous epithelial cells were counted from the entrance of the crypt opening to the beginning of the curve at the villous tip. Paneth cells were visualized by PAS/AB staining. Positive Paneth cells were counted per crypt. For counting of Paneth cells, 40 crypts were evaluated that were aligned along the longitudinal axis, and the lumen of the crypt can be visualized along its length. Counting was performed at $\times 20$ magnification. Quantification for Paneth and goblet cells was performed by a single investigator blinded to group and postnatal day.

\section{Immunohistochemistry}

The ileal tissues were immunostained using a rabbit anti-Ki67 (Thermo Fisher Scientific, Waltham, MA, Cat. No. RM-9106-S1) antibody followed by a biotinylated donkey anti-rabbit lgG, (Vector Labs, Burlingame, CA) and then by avidin-horseradish peroxidase conjugates (Vector Labs). Positive cells were visualized after incubation with 3,3'-diaminobenzidine (Sigma-Aldrich) for 
Effect of polyunsaturated fatty acids on postnatal ileum development...

P Singh et al.

Table 1. Gene list for quantitative RT-PCR analysis

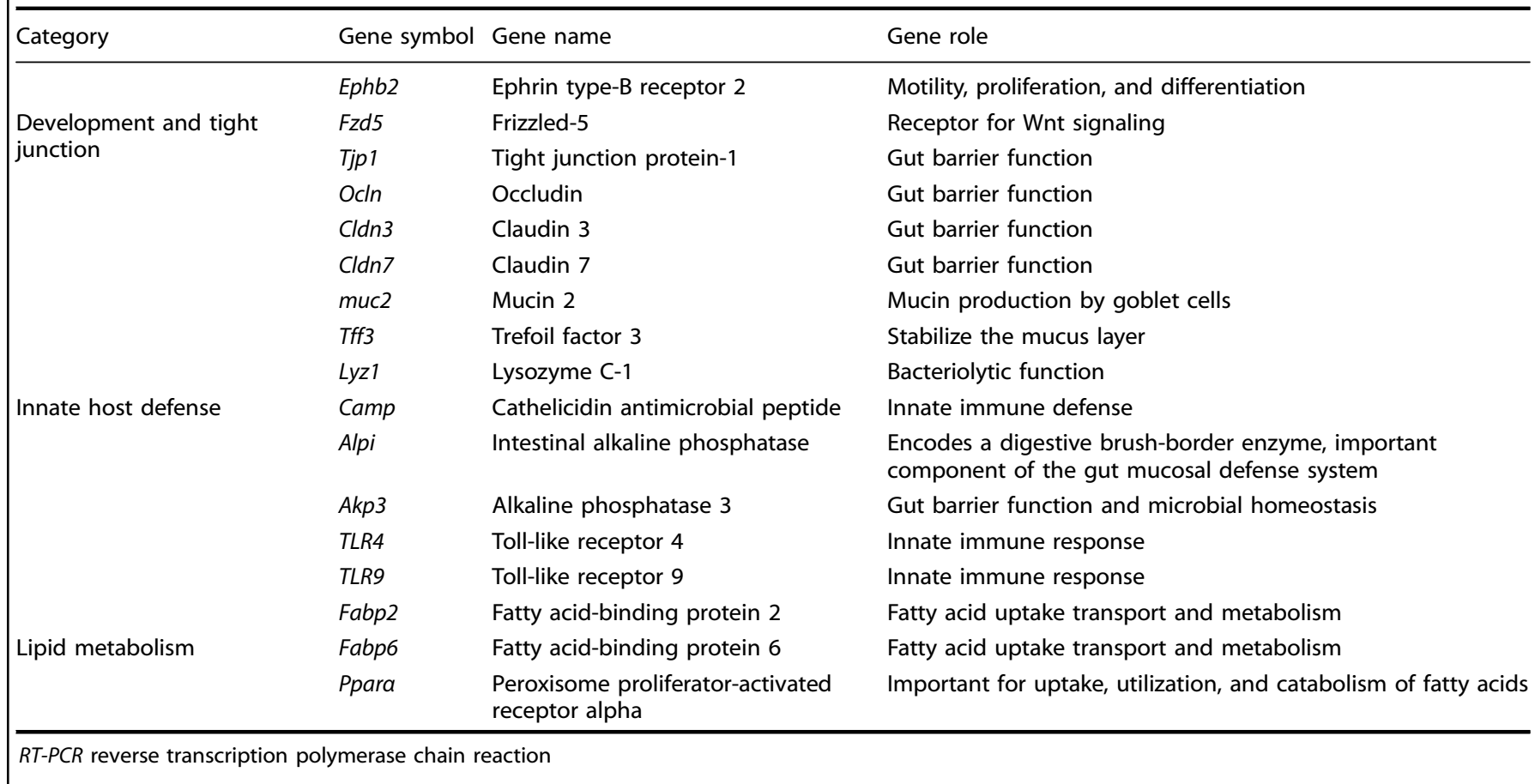

2-5 min and counterstained with hematoxylin. The total number of Ki67-positive cells was determined for each villous-crypt axis. Only well-oriented crypts with the epithelial layer on at least one side continuous with the villous epithelial layer were counted.

Reverse transcription-quantitative PCR (RT-qPCR)

Total RNA was isolated using the RNeasy Lipid Tissue Mini Kit (Qiagen, Germantown, MD) following the manufacturer's instructions. RNA concentration and purity were measured using a Nanodrop 2000 (Thermo Fisher Scientific, Waltham, MA). cDNA was synthesized with RT2 First Strand Kit (Qiagen). An RT2 profiler Custom PCR Array was used to simultaneously examine the mRNA levels of 17 genes characterizing cell differentiation, tight junctions, innate host defenses, and lipid metabolism (Table 1). The housekeeping genes HPRT, GAPDH, and Ywhaz and three controls including mouse genomic DNA control (MGDC) were included. RT-PCR was performed on an ABI Prism 7000 Sequence Detector (Applied Biosystems, Foster City, CA) using a standard cycling protocol.

Intestinal permeability

An intestinal permeability assay was performed as described previously. ${ }^{14}$ Briefly, mice were water-starved for $3.5 \mathrm{~h}$ and then gavaged with fluorescein isothiocyanate (FITC)-dextran 4000 (FD-4) (Sigma-Aldrich) in phosphate-buffered saline (PBS) at a concentration of $600 \mathrm{mg} / \mathrm{kg}$ body weight. Serum samples were collected after $3.5 \mathrm{~h}$ and diluted $1 / 100$ in PBS to measure the fluorescence intensity using a fluorospectrophotometer (Hitachi F-2000, Tokyo, Japan) with an excitation wavelength of $480 \mathrm{~nm}$ and an emission wavelength of $520 \mathrm{~nm}$. The concentration of FITC-dextran in serum samples was calculated using a known standard of serially diluted FITC-dextran. Serum samples of mice that did not receive any FITC-dextran were used to determine the background.

Statistical analysis

Distal ileum fatty acid levels were summarized by genotype and postnatal day using medians and interquartile range (IQR). Wilcoxon rank-sum test was used to compare the difference in fatty acids at each time point. For histological measurements of the distal ileum and intestinal permeability, statistical differences between more than two groups were analyzed by two-way analysis of variance (ANOVA) with Tukey's multiple comparison post hoc tests. Data are presented as bar graphs showing the mean along with standard deviation (SD).

RT-qPCR results were summarized and displayed as median fold change $( \pm I Q R)$ of fat-1 gene expression relative to WT for each postnatal day. Fold changes were calculated using the $2^{\wedge}-\Delta \Delta \mathrm{Ct}$ method. A median fold change $>1$ represents upregulation and a value $<1$ represents downregulation. The $\Delta \mathrm{Ct}$ values for each gene of interest were used to determine statistically significant differences in gene expression between groups. A two-way ANOVA was performed to evaluate differences in gene expression in the fat-1 group relative to the WT group over time. If the result of the two-way ANOVA test was statistically significant, then Tukey's multiple comparison post hoc tests were used to determine which genes were differentially expressed between the two groups at each time point. To determine the relative contribution of specific fatty acid levels on gene expression, a correlation matrix was constructed using all fatty acid levels and genes studied. Significant correlations were used to construct a correlation network.

All analyses were performed using the R software (version 3.5.1, $\mathrm{R}$ Core Team, Vienna, Austria, 2018) within RStudio (Version 1.1.453, RStudio, Inc.) using the tidyverse (Wickham 2017), PMCMRplus (Pohlert 2018), ggcorrplot (Kassambara 2016), RColorBrewer (Neuwirth 2014), and igraph (Csardi and Nepusz 2006) packages. Results were considered significant at $p<0.05$.

\section{RESULTS}

Distal ileum fatty acid profiles in WT versus fat-1 transgenic mice On day 3 , the distal ileum of fat- 1 versus WT mice had a 10- and 26 -fold increase in the $n-3$ fatty acids a-linolenic acid (ALA) and eicosapentaenoic acid (EPA), respectively (Table 2). Conversely, there was a two-fold reduction in the n- 6 fatty acid AA in fat- 1 mice relative to the WT group. In addition to the individual fatty 


\begin{tabular}{|c|c|c|c|c|c|c|c|c|c|c|c|c|}
\hline \multirow[t]{2}{*}{ Fatty acid group } & \multicolumn{4}{|l|}{ Day 3} & \multicolumn{4}{|l|}{ Day 14} & \multicolumn{4}{|l|}{ Day 28} \\
\hline & Median & IQR & Median & IQR & Median & IQR & Median & IQR & Median & IQR & Median & IQR \\
\hline \multicolumn{13}{|l|}{$n-3$} \\
\hline EPA & 0.05 & $0.04,0.05$ & 1.3 & $1.1,2.2$ & 0.07 & $0.06,0.07$ & 2.2 & $1.9,2.4$ & 0.04 & $0.03,0.04$ & 2.1 & $1.5,2.4$ \\
\hline DHA & 4.2 & $3.6,4.8$ & 3.7 & $3.5,5.0$ & 3.4 & $3.0,3.5$ & 6.2 & $5.9,6.9$ & 1.1 & $0.9,1.3$ & 2.2 & $1.8,2.3$ \\
\hline \multicolumn{13}{|l|}{$n-6$} \\
\hline $\mathrm{LA}$ & 14.0 & $13.5,14.3$ & 16.0 & $15.5,16.0$ & 15.0 & $15.0,15.3$ & 15.5 & $14.8,16.3$ & 23.0 & $21.0,25.0$ & 24.0 & $23.0,25.3$ \\
\hline GLA & 0.3 & $0.2,0.3$ & 0.4 & $0.3,0.4$ & 0.1 & $0.09,0.1$ & 0.07 & $0.05,0.09$ & 0.1 & $0.1,0.2$ & 0.1 & $0.1,0.1$ \\
\hline
\end{tabular}

acids highlighted, when compared to WT mice, the fat-1 mice had a greater $n-3: n-6$ ratio. As the mice increased in age, the genotype to fatty acid relationships persisted with the addition of a two-fold increase in DHA level in fat- 1 mice on day 14 and 28 compared to WT mice and an increasing fold change in the $n$ 3:n-6 ratio from 1.6 on day 3 to 3.5 and 4.4 on days 14 and 28, respectively. During the neonatal period from day 3 to day 14, DHA levels declined by $20 \%$ in the WT group. In contrast, in the fat-1 group, DHA levels increased by almost $70 \%$. Both groups demonstrated the lowest DHA levels at day 28, though the fat-1 group remained two-fold higher compared to WT mice. The complete ileum fatty acid profiles of fat- 1 and WT mice on days 3,14 , and 28 are summarized in Supplemental Tables S4, S5, and S6, respectively.

\section{Histology}

Villous height, crypt depth, and muscular thickness. Villous height, crypt depth, and muscular thickness were quantified using H\&Estained distal ileal sections (Fig. 1a). The average villous height was unchanged across days 3,14 , and 28 for WT mice $(183.6 \pm 26.3 \mu \mathrm{m}$, $197.3 \pm 16.2 \mu \mathrm{m}$, and $169.2 \pm 37.9 \mu \mathrm{m}$, respectively; Fig. $1 \mathrm{~b})$. In contrast to WT mice, the villous height of fat-1 mice decreased over time relative to the day 3 height with values of $224.9 \pm$ $36.8 \mu \mathrm{m}, 176.9 \pm 13.6 \mu \mathrm{m}$ and $150.2 \pm 12.7 \mu \mathrm{m}$ on days 3,14 , and 28 , respectively (day 28 versus day $3, p<0.002$ ). Crypt depth on day 3 for both genotypes appeared as shallow invaginations that become deeper and well organized on days 14 and 28 (Fig. 1a). In WT mice, the crypt depth increased over time from $24.3 \pm 3.3 \mu \mathrm{m}$ on day 3 to $49.9 \pm 6.0 \mu \mathrm{m}$ on day 28 ( $p<0.0001$, Fig. $1 \mathrm{c})$. Similarly, in fat-1 mice, the crypt depth increased from $23.3 \pm 3.3 \mu \mathrm{m}$ on day 3 to $43.5 \pm 3.5 \mu \mathrm{m}$ on day $28 \quad(p<0.0001$, Fig. 1c). Muscular thickness increased from day $3(21.4 \pm 4.0 \mu \mathrm{m}$ for WT; $22.6 \pm$ $2.7 \mu \mathrm{m}$ for fat-1) to day $28(41.7 \pm 5.7 \mu \mathrm{m}$ for $\mathrm{WT} ; 35.08 \pm 4.1 \mu \mathrm{m}$ for fat-1) in both groups ( $p<0.0001$, Fig. 1d). Overall, although there was an age dependency in villous height, crypt depth, and muscular thickness, there were no significant differences for these parameters between WT and fat-1 transgenic mice (Fig. 1).

Goblet and Paneth cells. The number of AB-positive goblet cells/ 100 epithelial cells in the intestinal villi were analyzed using $A B$ staining (Fig. 2a). We show a genotype-day interaction $(p=0.013)$ with decreased numbers of goblet cells containing acidic mucin in fat-1 compared to WT mice at each day $(p<0.0001, p=0.17$, and $p<0.0001$, respectively) (Fig. 2b). The number of goblet cells $/ 100$ epithelial cells did not change over time in WT mice, while in fat-1 mice there was a decline in goblet cells from day 14 to day 28 $(p=0.019)$.

For the neutral mucins, we also see a genotype-day interaction $(p<0.0001)$ with decreased number of PAS-stained cells on day 3 $(p<0.0001)$ and $28(p<0.001)$ in fat 1 compared to WT mice (Supplemental Figure S2). In contrast to the acidic mucins, both groups showed a decline in neutral mucins over time.

Paneth cells at the base of crypts were visualized using PAS/AB staining (Fig. 3a). The appearance of Paneth cells was age dependent. The number of Paneth cells/crypt were significantly greater on day 28 compared to day 14 in both groups of mice (1.8-2.0 \pm 0.2 versus $3.3-3.5 \pm 0.2, p<0.0001)$, while no Paneth cells were identifiable on day 3 . We did not observe any differences in the number of Paneth cells/crypt between fat-1 transgenic and WT mice (Fig. 3b).

Crypt cell proliferation. Crypt cell proliferation was quantified by Ki67 staining (Supplemental Figure S3). The number of Ki67positive cells increased significantly over time within each genotype $(p<0.0001)$, with no differences across the two groups.

Effect of fatty acid profiles on gene expression. The impact of fatty acid profiles on intestinal development was studied by quantifying the differences in the level of gene expression between the two genotypes across age. Genes were selected to represent several categories of intestinal development and function including cell differentiation and tight junction formation, innate host defenses, and lipid metabolism (Fig. 4).

Cell differentiation and tight junction. Fzd5 is a receptor for the Wnt signaling pathway and EphB2 is required for cell proliferation, differentiation, and cell migration. We found higher expression level of Fzd5 (3.2-fold) in 3-day-old fat-1 mice $(p<0.0001)$ relative to the WT group on the same postnatal day. The expression level of EphB2 was also higher in fat-1 mice on day 3 and 28 (2.2-fold, 
a
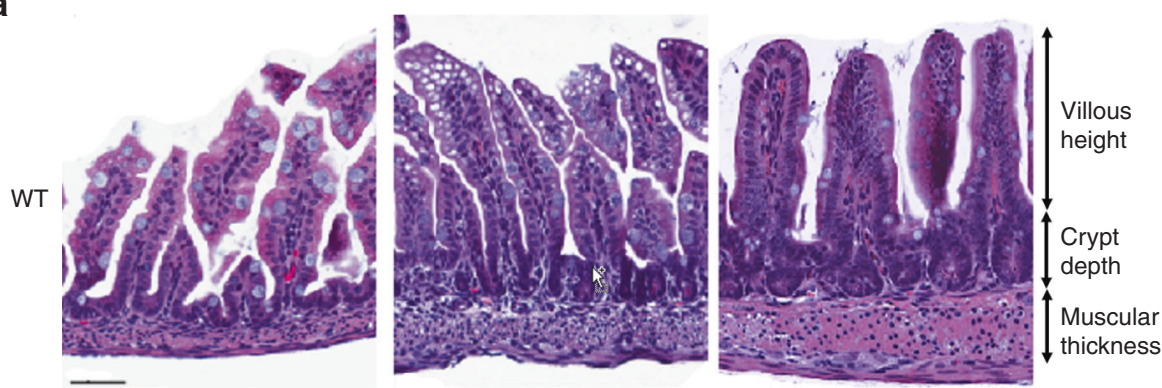

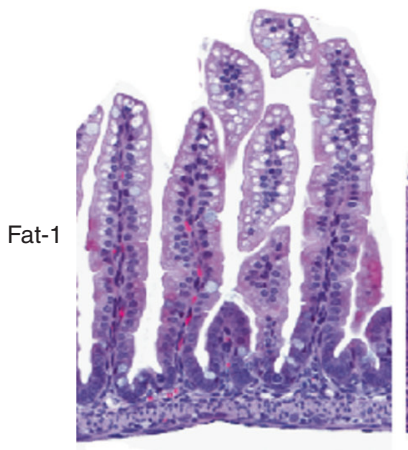

Day 3

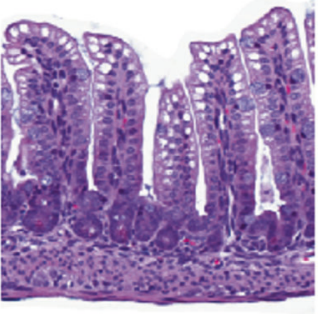

14

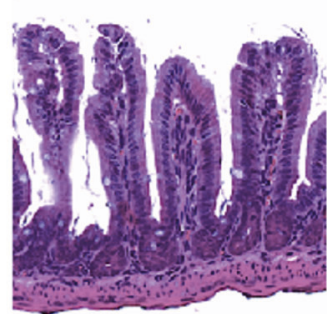

28 b

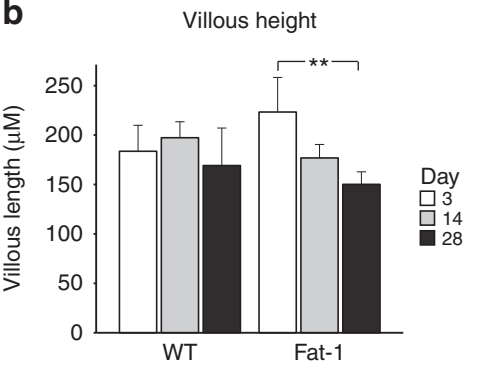

C Crypt depth

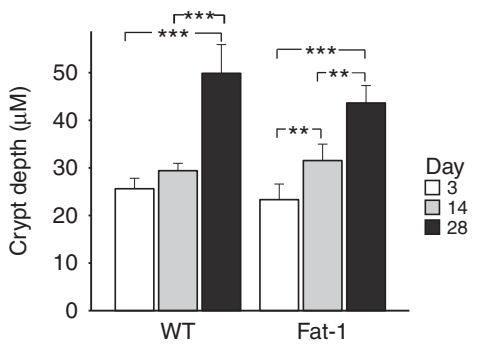

d

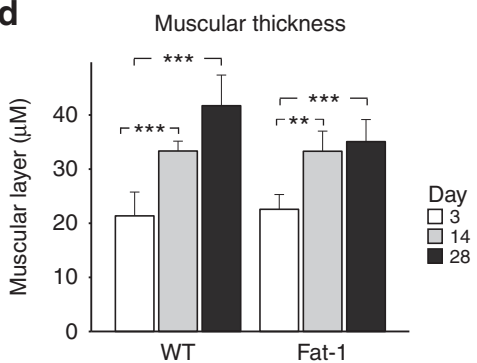

Fig. 1 Effect of genotype-induced fatty acids profiles on intestinal morphology during postnatal growth. Hematoxylin and eosin-stained sections of distal ileal segments of the small intestine from days 3, 14, and 28 in wild-type and fat-1 transgenic mice. a Representative images are shown for the indicated ages. Bar graphs show villous height $(n=3-6)(\mathbf{b})$, crypt depth $(n=4-6)(\mathbf{c})$, and muscular layer thickness (d) $(n=$ $4-7)$. Results shown as mean \pm SD and $p$ values $<0.05$ were considered statistically significant $\left({ }^{* *} p<0.001\right.$ and $\left.{ }^{* * *} p<0.0001\right)$

$p<0.0001$ and 1.7 -fold $(p<0.01)$, respectively). Compared to WT mice, fat-1 mice showed reduced expression level of claudin3 (Cldn3) and claudin7 (Cldn7), 1.9-fold and 3.0-fold, respectively, on day $3(p<0.0001)$ and reduced expression of Cldn3 on day 28 by 1.4-fold $(p<0.05)$.

Innate host defense. The expression level of Camp, a gene that encodes for a secreted peptide that has a pleiotropic function such as antimicrobial and immunomodulatory effect, ${ }^{15}$ was lower in fat- 1 transgenic mice compared to WT on day 3 by 3.6 -fold ( $p<$ 0.0001 ). The expression level of $T L R 9$ was significantly lower in fat1 mice compared to WT with a 2.5 -fold downregulation on day 3 and 1.9-fold downregulation on day $28(p<0.05)$. No difference in TLR9 expression was found on day 14 between the two groups or TLR4 at any time. The expression level of muc2 and Tff3 was reduced by $>2.0$-fold in fat- 1 mice on day $14(p<0.05)$.

Lipid metabolism. Membrane-bound fatty acid-binding proteins (FABPs) are essential for fatty acid uptake, transport, and metabolism. Alterations in lipid metabolism regulate the expression of FABPs at the transcription level. ${ }^{16}$ FABP2 is expressed throughout the small intestine, and FABP6 is shown explicitly in the ileal region of the small intestine. ${ }^{17}$ Our data indicate that the expression level of Fabp2 in fat-1 mice was increased by 2.9-fold and 1.8-fold on days 3 and 28 respectively, compared to WT mice $(p<0.0001$ and $p<0.001$, respectively). Similarly, the expression level of Fabp6 was significantly increased in fat-1 mice on day 3 (3.5-fold, $p<0.05)$ while no significant differences were found on days 14 and 28 . Additionally, fat-1 mice showed significant reductions in the expression level of Ppara on day 3 (2.0-fold, $p<$ 0.001 ) but not on day 14 or 28.

Intestinal permeability. The observation of decreased Cldn3 and Cldn7 at day 3 in fat- 1 versus WT mice led us to further examine a functional assay in intestinal permeability using FD-4 (Fig. 5). The percentage of leak in WT mice did not significantly change from day 3 to day 28 . In contrast, fat- 1 mice showed a significant reduction in percent leak from day 3 to day $28(0.9 \%$ versus $0.4 \%$, $p<0.001)$. The main effect of genotype was marginally significant at $p=0.048$. Additionally, the interaction effect of time and genotype was significant $(p<0.01)$, indicating that the genotype effect was greater in the fat- 1 versus WT mouse in percentage of leak over time.

Fatty acid-gene expression correlation network. We created a correlation network to determine the relative contribution of specific fatty acid on gene expression (Fig. 6). The network naturally divided into two sub-networks with DHA the primary fatty acid in the first and ALA the primary fatty acid in the second. DHA appears to have a direct influence on the expression level of various genes related to tight junction and Ppara. ALA has a direct link in the expression of genes related to cellular development (EphB2 and $F z d 5)$ and the Fabp, which then were correlated with the expression of other host defense genes, such as muc2, Tff3, and Lyz1. 
a
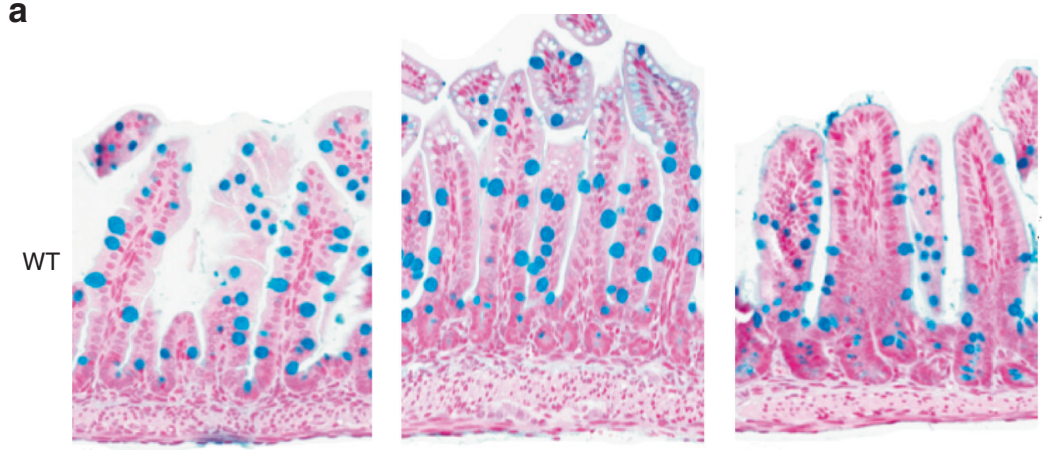

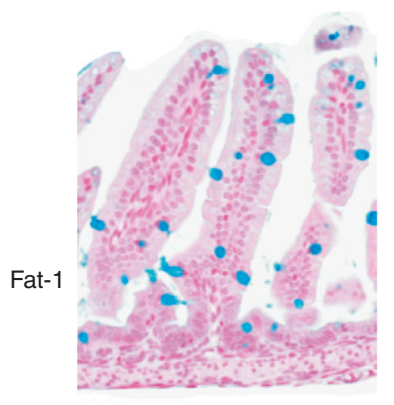

Day 3

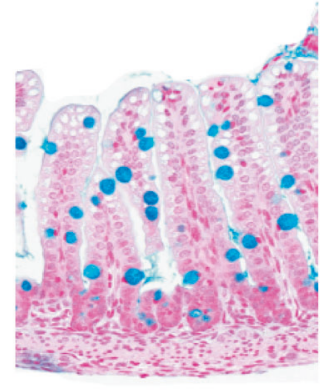

14
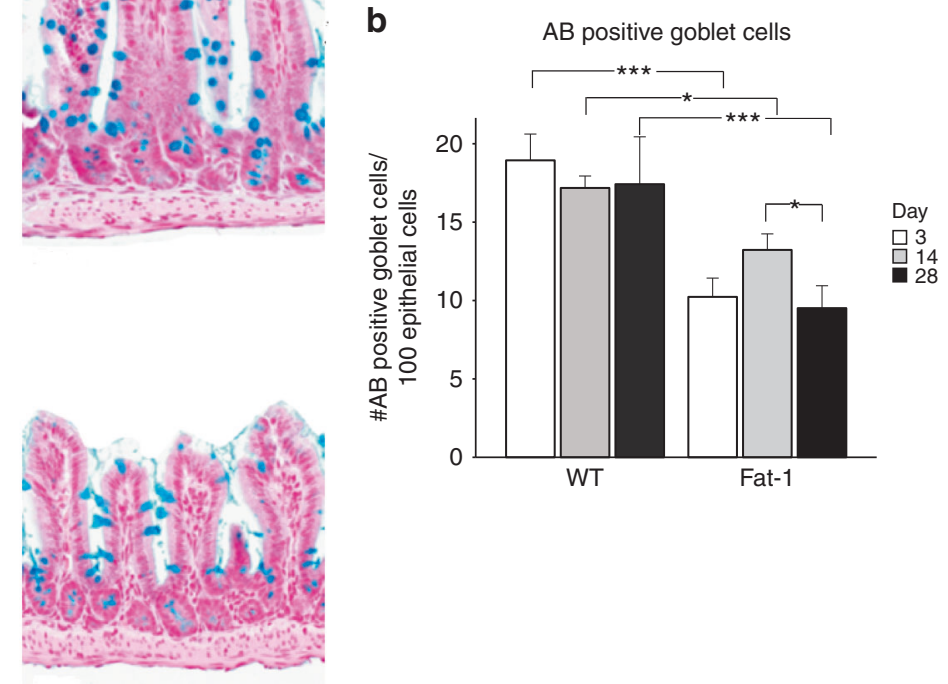

Fig. 2 Effect of genotype-induced fatty acids profiles on Alcian Blue (AB)-positive goblet cells. Ileal tissue sections from fat-1 transgenic and wild-type mice were stained with $A B$ to mark goblet cells filled with acidic mucins and counterstained with eosin. a Representative images are shown for the indicated ages. b The graph shows the number of AB-stained cells per 100 epithelial cells $(n=4-5)$. Results shown as mean \pm SD and $p$ values $<0.05$ were considered statistically significant $\left({ }^{*} p<0.05,{ }^{* *} p<0.0001\right)$

a
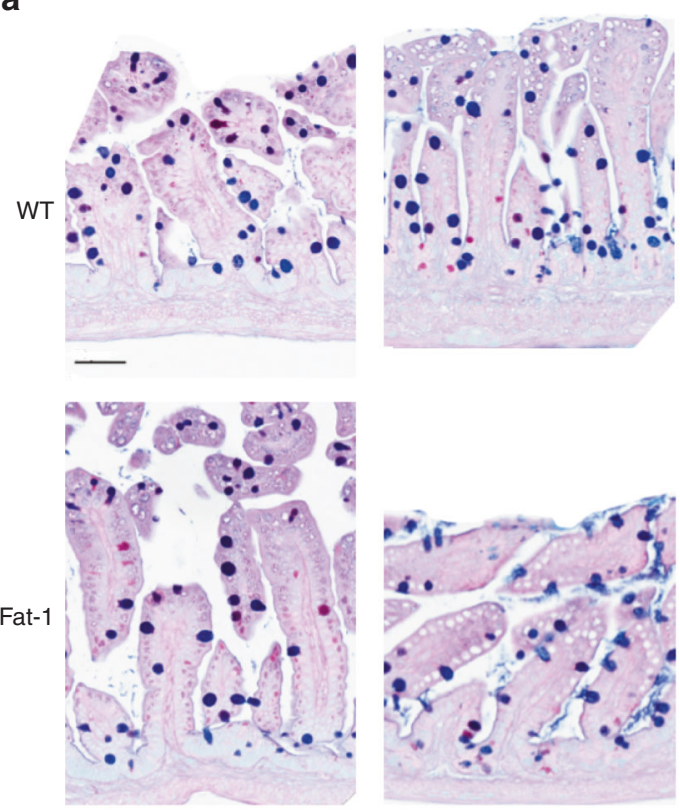

Day 3

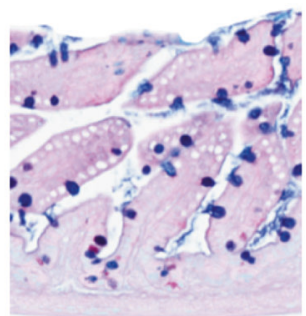

14

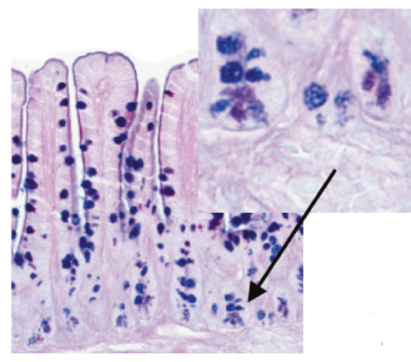

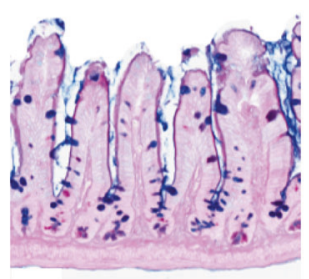

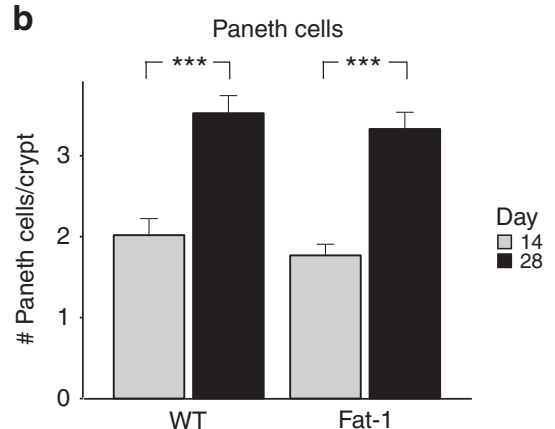

Fig. 3 Effect of genotype-induced fatty acids profiles on Paneth cells. lleal sections from fat-1 transgenic and wild-type mice were stained with periodic acid-Schiff (PAS)/Alcian Blue (AB) to mark Paneth cells and counterstained with eosin. a Representative slides are shown for the indicated ages. Arrow points to the specific granular PAS/AB staining of Paneth cells. b Graphs show the number of Paneth cells per crypt ( $n=$ $4-5)$. Columns show the mean \pm SD and $p$ values $<0.05$ were considered statistically significant $(* * * p<0.0001)$ 

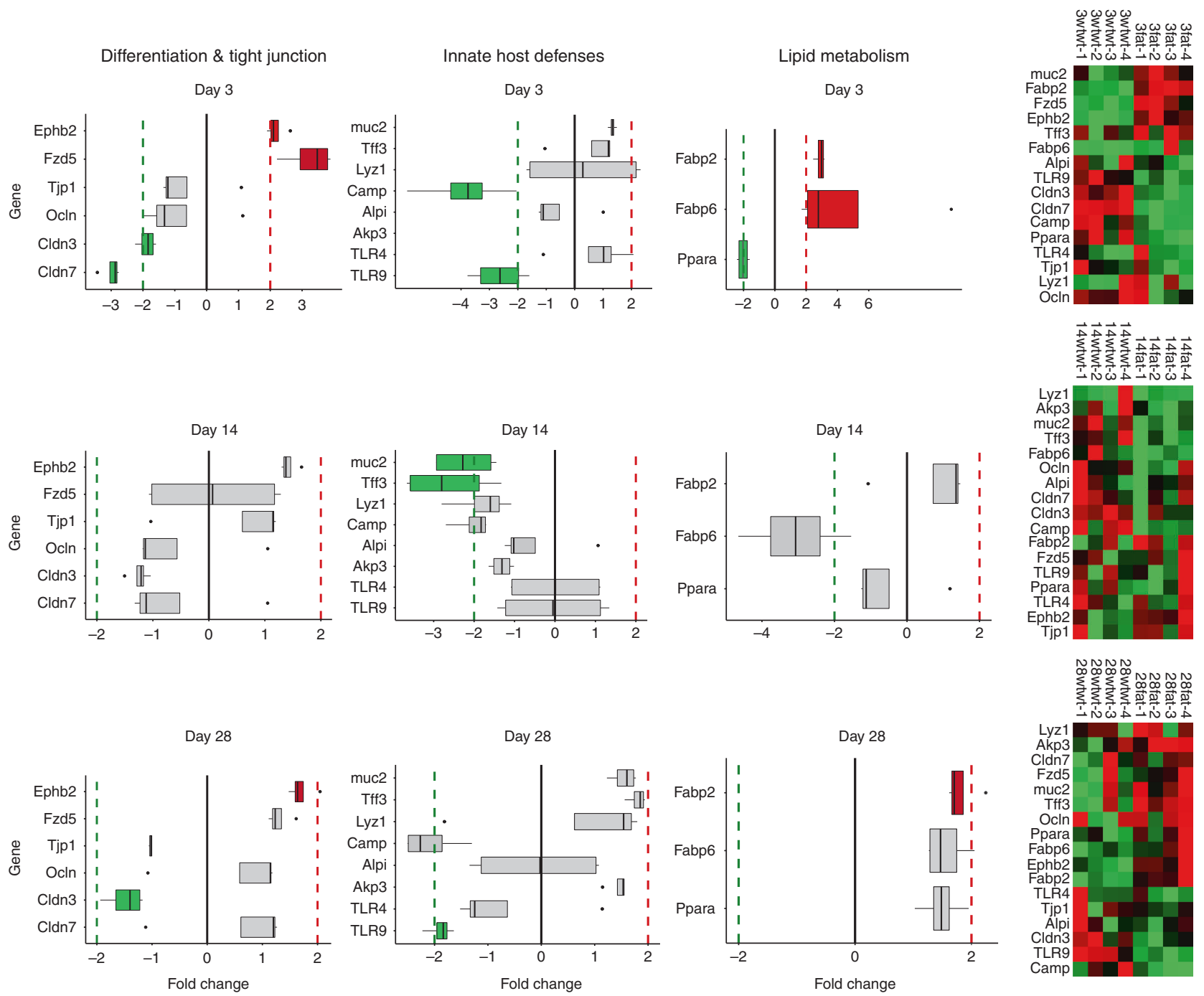

Fig. 4 Box and whisker plots of median fold change in gene expression. Results depicted across time in postnatal days and grouped by general functional category. Expression of the genes in fat-1 mice were normalized as fold change to the baseline of wild-type controls. Red indicates a statistically significant upregulation in expression. Green indicates a statistically significant downregulation in expression. Gray boxes indicate no statistically significant difference. Black dots are values outside of the 25-75th percentile of a boxplot. For each postnatal day, a heatmap on the right summarizes the fold change for each gene ( $y$ axis) for each mouse clustered by genotype ( $x$ axis)

\section{DISCUSSION}

Our data support the hypothesis that fatty acid profiles early in development modulate intestinal gene expression in formative domains, such as cell differentiation, tight junctions, other innate host defenses, and lipid metabolism. The fat-1 transgenic mouse allowed us to isolate the effect of fatty acid profiles on gut development without the potential confounding factor of dietary composition. By comparing the two genotypes, we were able to mimic fatty acid profiles observed in the postnatal period in preterm infants receiving the current standard of nutritional care to those who receive fish-oil-based nutrition with the goal of reducing early postnatal DHA deficits experienced by preterm infants. As predicted, the fat-1 group relative to the WT group had significantly higher levels of ALA, an initial precursor of the n-3 pathway as well as the downstream products such as EPA and DHA by day 14 . This was accompanied by a reduction in the $n-6$ fatty acid AA, indicating a conversion of $n-6$ to $n-3$ fatty acids, and an overall increase in the $n-3: n-6$ ratio. With these divergent fatty acid levels, differential expression of key genes involved in intestinal development was seen across time between the two groups. During the preweaning periods (days 3 and 14), differential gene expression was most evident on day 3 with an upregulation in markers of cell differentiation and FABPs and a downregulation in Ppara, Camp, TLR9, and genes related to tight junctions. Tff3 and muc2 were downregulated on day 14. This indicates that intestinal development and function in the preterm infant is highly vulnerable to the specific composition of nutrient delivery.

Increased expression of Fzd5 in fat-1 mice suggests that conversion of $n-6$ to $n-3$ fatty acids modulate the Wnt signaling pathways by regulating the expression of $F z d 5$ at an early phase of life. EphB2, Eph receptor tyrosine kinase, was also expressed at higher levels in fat-1 mice possibly due to Wht signaling.

FABPs are very important for lipid-mediated biological process and in metabolic and immune response by regulating lipid signals. $^{17}$ Intestinal FABP (I-FABP), also known as FABP2, is expressed by mature enterocytes and is released in circulation as a result of enterocyte injury and thus used as an early biomarker for intestinal damage. ${ }^{18}$ In a rat model of ischemia/ reperfusion, n-3 LCPUFAs significantly reduced plasma levels of I-FABP while increasing its concentration in the intestinal mucosa and provided protection against intestinal injury. ${ }^{19,20}$ Our results showed increased levels of both Fabp2 and 6 in fat-1 transgenic mice compared to WT mice on day 3 , suggesting that $n-3$ fatty 


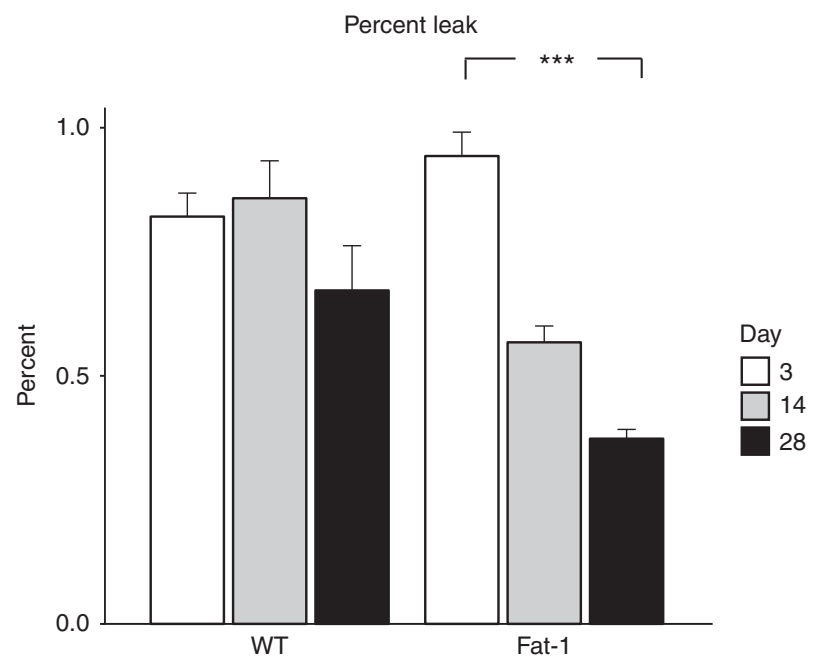

Fig. 5 Effect of genotype-induced fatty acids profiles on intestinal permeability. Wild-type and fat-1 mice of different ages (days 3, 14, and 28) were administered FITC-dextran 4000 (FD-4) by oral gavage and data are shown as percentage of leak. Combined results from independent experiments are shown as a bar graph with mean \pm SD ( $n=8-32$ per group). $p$ values $<0.05$ were considered statistically significant $(* * * p<0.0001)$

acids modulate the expression of these two genes. In the absence of injury, upregulation of these genes could be correlated with an increased turnover of mature enterocytes in fat-1 transgenic mice.

CAMP and TLR9 play a role in neutralizing bacteria and/or mounting an inflammatory response. ${ }^{21-24}$ CAMP is expressed by neutrophils, macrophages, and intestinal epithelial cells and possesses antimicrobial property. Short chain fatty acids induce the expression of cathelicidine LL-37 mRNA in human colonic epithelial cells. ${ }^{25}$ Besides its antimicrobial properties, CAMP is vital for intestinal homeostasis ${ }^{21}$ although the physiological relationship between decreased levels of CAMP level and gut development and maturation has yet to be fully elucidated. With regard to the role of TLR9 in regulating inflammatory responses in the gut, previous studies have shown conflicting results using the dextran sodium sulfate (DSS)-induced colitis model. ${ }^{23,24}$ Rachmilewitz et al. showed no difference in the severity of DSS-induced colitis between WT and TLR9-deficient mice. ${ }^{22}$ In contrast, Lee et al. and Rose et al. showed enhanced susceptibility of TLR9-deficient mice to DSS-induced colitis. ${ }^{23,26}$ Moreover, TLR9-deficient mice showed enhanced severity of NEC, while CpG-DNA, a ligand that activates $T L R 9$, administration decreased severity. ${ }^{4}$ These latter data suggest a protective role for TLR9 in these models of intestinal injury. Camp and TLR9 genes were downregulated in fat-1 compared to WT mice, indicating that the conversion of $n-6$ to $n-3$ LCPUFAs directs the mouse intestine to a reduced inflammatory state. Whether this is protective or predisposes the host to an inadequate response to infection or injury remains to be determined.

In a term infant, the intestinal barrier rapidly develops over days after the introduction to enteral feedings. In a preterm infant, intestinal closure is delayed and this is thought to be one contributing factor to the increased vulnerability to intestinal injury and NEC. ${ }^{27,28}$ Several in vitro studies have presented contradictory results about the role of LCPUFAs in tight junction integrity and gut permeability. ${ }^{29-31}$ In the present study, fat-1 mice showed statistically significant reduced expression of Cldn3 and 7 but did not show any changes in the expression of Ocln and Tjp-1. Our results are in agreement with Beguin et al., who reported that $n-6$ but not n-3 fatty acids resulted in significant changes on Ocln, Tjp-1, and intestinal permeability. ${ }^{31}$ Moreover, they showed that DHA when used at a very high concentration induced substantial changes in Tjp-1 expression accompanied

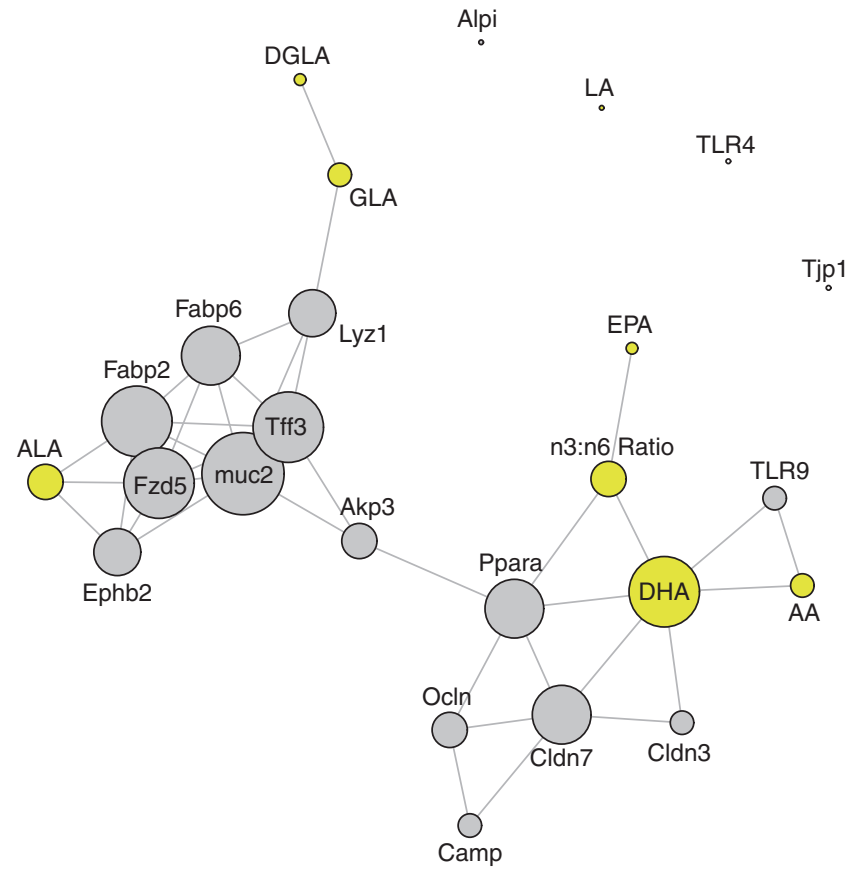

Fig. 6 Fatty acid-gene correlation network. Only statistically significant $(p<0.05)$ correlations $(r>0.58)$ are depicted in this network. Yellow nodes represent fatty acids. Gray nodes represent genes. The size of the node is proportional to the number of edges interacting with the node

with a minimal effect on permeability. Our data suggest that the impact of LCPUFAs on the expression of different tight junction genes is age dependent and this effect could vary for individual tight junction proteins. Our permeability assay results confirm that intestinal permeability may not be dependent solely on the expression of known tight junction genes. Factors such as other proteins involved in the formation of tight junctions, the composition of gut microbiota, epithelial damage, and changes in mucus layer thickness can all alter intestinal permeability. Our results do suggest that conversion of $n-6$ to $n-3$ can reduce western diet-induced increased intestinal permeability. ${ }^{32}$

In contrast to the multiple differences in gene expression observed between fat-1 and WT mice, there were minimal differences in the actual morphological structure and cell populations of the ileum between the two groups. Both groups did show the expected developmental change in these elements over time. The one factor that did show a difference was the presence of goblet cells. Fat-1 mice relative to WT mice of the same age had a reduced number of goblet cells. Although the DHA-derived terminal metabolite Resolvin D1 is known to increase ocular mucin secretion from goblet cells, the role of DHA and its metabolites on intestinal goblet cells is unknown. ${ }^{33}$ Metabolites derived from AA impact goblet cell differentiation in airway epithelial cells. ${ }^{34}$ Although AA metabolites were not shown to increase mucin production in intestinal goblet cells of a rabbit, the absolute quantification of the number of goblet cells was not performed in this in vitro study. ${ }^{35}$ It is possible that the reduction in goblet cell number in fat-1 mice was a result of declining AA levels, which is a known compensatory consequence of $n-3$ enrichment. $^{36}$

A limitation of our study design was the inability to assign the gene expression changes to a single fatty acid. The correlation network revealed the influence of both ALA and DHA that infers to the importance of multiple fatty acids and their interactions to exert broad biological effects, not just one. Additional studies modulating a single fatty acid will still be important to understand 
and will be a natural follow-up to this study. We did not study the mediating effect of the microbiome in fatty acid-induced changes in gene expression. It is likely the microbiome plays some role in these processes. Recent studies have shown the impact of $n-3$ PUFAs on gut microbiota. ${ }^{37}$ Additional studies are needed to investigate the impact of PUFA-driven changes in the microbiome and subsequent intestinal development during the neonatal period.

The strengths of the study design were the ability to mimic postnatal fatty acid levels observed in preterm infants and isolate the effect of $n-6$ versus $n-3$ dominant fatty acid profiles on intestinal development using the fat- 1 transgenic model. This design was of particular interest given the clinical motivation to increase the $n-3$ delivery to preterm infants to overcome the early postnatal deficit in DHA that occurs. The fatty acid profiles in the fat-1 mice resemble those observed in preterm infants when $n-3$ enriched nutrition is provided - an increase in DHA and EPA, with a concomitant decrease in $A A^{36}$ We can conclude that diet not only changes systemic fatty acid profiles but also affect the gene expression patterns in the developing gut. Though some of these changes in expression and function might have desirable effects such as augmenting cell differentiation and intestinal permeability, other changes may have mixed effects such as downregulation of goblet cell formation and innate immune host defenses. Excessive inflammation is not desirable, but inhibiting inflammation may not be appropriate either in specific clinical situations. $^{38}$ The impact of these host changes that are primed by early nutrition will need to be evaluated in models of intestinal stress and injury.

\section{CONCLUSIONS}

The fat- 1 transgenic mouse model provides a valuable tool to study the effect of n-3-enriched diets on gut development. This model allows for well-controlled studies without the interference of potential dietary confounding factors. Our data confirm that LCPUFAs act as a regulator for early gut development demonstrating a broad range of effects on gene expression, goblet cell number, and intestinal permeability. Future experiments using this experimental model in induced intestinal injury will be required to determine the role of different fatty acid profiles in disease risk and pathogenesis.

\section{ACKNOWLEDGEMENTS}

We are grateful to Dr. Jing X. Kang (Massachusetts General Hospital, Boston, MA) for providing us the fat-1 transgenic mice. Our study was supported by Charles $\mathrm{H}$. and Judy Hood Family Infant Health Research Program and NIH R01 DK104346.

\section{AUTHOR CONTRIBUTIONS}

Substantial contributions to conception and design, acquisition of data, or analysis and interpretation of data: all the authors. Drafting the article or revising it critically for important intellectual content: P.S., C.R.M., S.D.F. and G.P. Final approval of the version to be published. P.S., C.R.M., and S.D.F.

\section{ADDITIONAL INFORMATION}

The online version of this article (https://doi.org/10.1038/s41390-019-0284-0) contains supplementary material, which is available to authorized users.

Competing interests: The authors declare no competing interests.

Publisher's note: Springer Nature remains neutral with regard to jurisdictional claims in published maps and institutional affiliations.

\section{REFERENCES}

1. Neu, J. \& Walker, W. A. Necrotizing enterocolitis. N. Engl. J. Med. 364, 255-264 (2011).

2. Lin, P. W. \& Stoll, B. J. Necrotising enterocolitis. Lancet 368, 1271-1283 (2006).

3. Tanner, S. M. et al. Pathogenesis of necrotizing enterocolitis: modeling the innate immune response. Am. J. Pathol. 185, 4-16 (2015).

4. Gribar, S. C. et al. Reciprocal expression and signaling of TLR4 and TLR9 in the pathogenesis and treatment of necrotizing enterocolitis. J. Immunol. 182, 636-646 (2009).

5. Martin, C. R. et al. Decreased postnatal docosahexaenoic and arachidonic acid blood levels in premature infants are associated with neonatal morbidities. $J$. Pediatr. 159, 743-749 (2011). e1-2.

6. Löfqvist, C. A. et al. Association of retinopathy of prematurity with low levels of arachidonic acid: a secondary analysis of a randomized clinical trial. JAMA Ophthalmol. 136, 271-277 (2018).

7. Marion-Letellier, R., Savoye, G., Beck, P. L., Panaccione, R. \& Ghosh, S. Polyunsaturated fatty acids in inflammatory bowel diseases: a reappraisal of effects and therapeutic approaches. Inflamm. Bowel Dis. 19, 650-661 (2013).

8. Wijendran, V. et al. Long-chain polyunsaturated fatty acids attenuate the IL-1 $\beta$ induced proinflammatory response in human fetal intestinal epithelial cells. Pediatr. Res. 78, 626-633 (2015).

9. Ohtsuka, Y. et al. $\omega-3$ fatty acids attenuate mucosal inflammation in premature rat pups. J. Pediatr. Surg. 46, 489-495 (2011).

10. Lu, J., Jilling, T., Li, D. \& Caplan, M. S. Polyunsaturated fatty acid supplementation alters proinflammatory gene expression and reduces the incidence of necrotizing enterocolitis in a neonatal rat model. Pediatr. Res. 61, 427-432 (2007).

11. Kang, J. X., Wang, J., Wu, L. \& Kang, Z. B. Transgenic mice: fat-1 mice convert n-6 to n-3 fatty acids. Nature 427, 504 (2004).

12. McElroy, S. J. \& Weitkamp, J.-H. Innate immunity in the small intestine of the preterm infant. Neoreviews 12, e517-e526 (2011).

13. Freedman, S. D. et al. A membrane lipid imbalance plays a role in the phenotypic expression of cystic fibrosis in $\mathrm{cftr}(-/-)$ mice. Proc. Natl. Acad. Sci. USA 96, 13995-14000 (1999).

14. Economopoulos, K. P. et al. Prevention of antibiotic-associated metabolic syndrome in mice by intestinal alkaline phosphatase. Diabetes Obes. Metab. 18, 519-527 (2016).

15. Coorens, M., Scheenstra, M. R., Veldhuizen, E. J. A. \& Haagsman, H. P. Interspecies cathelicidin comparison reveals divergence in antimicrobial activity, TLR modulation, chemokine induction and regulation of phagocytosis. Sci. Rep. 7, 40874 (2017).

16. MMAL, Pelsers, Hermens, W. T. \& Glatz, J. F. C. Fatty acid-binding proteins as plasma markers of tissue injury. Clin. Chim. Acta 352, 15-35 (2005).

17. Furuhashi, M. \& Hotamisligil, G. S. Fatty acid-binding proteins: role in metabolic diseases and potential as drug targets. Nat. Rev. Drug Discov. 7, 489-503 (2008).

18. Ng, E. W. Y. et al. Gut-associated biomarkers L-FABP, I-FABP, and TFF3 and LIT score for diagnosis of surgical necrotizing enterocolitis in preterm infants. Ann. Surg. 258, 1111-1118 (2013).

19. Li, Y., Wang, X., Li, N. \& Li, J. The study of n-3PUFAs protecting the intestinal barrier in rat HS/R model. Lipids Health Dis. 13, 146 (2014).

20. Wang, X., Pan, L., Lu, J., Li, N. \& Li, J. N-3 PUFAs attenuate ischemia/reperfusion induced intestinal barrier injury by activating I-FABP-PPARY pathway. Clin. Nutr. 31, 951-957 (2012).

21. Otte, J.-M. et al. Effects of the cathelicidin LL-37 on intestinal epithelial barrier integrity. Regul. Pept. 156, 104-117 (2009).

22. Rachmilewitz, D. et al. Toll-like receptor 9 signaling mediates the antiinflammatory effects of probiotics in murine experimental colitis. Gastroenterology 126, 520-528 (2004).

23. li, W. A. R., Sakamoto, K. \& Leifer, C. A. TLR9 is important for protection against intestinal damage and for intestinal repair. Sci. Rep. 2, 574 (2012).

24. Lee, J., Rachmilewitz, D. \& Raz, E. Homeostatic effects of TLR9 signaling in experimental colitis. Ann. NY Acad. Sci. 1072, 351-355 (2006).

25. Jiang, W. et al. Differential regulation of human cathelicidin LL-37 by free fatty acids and their analogs. Peptides 50, 129-138 (2013).

26. Lee, J. et al. Maintenance of colonic homeostasis by distinctive apical TLR9 signalling in intestinal epithelial cells. Nat. Cell Biol. 8, 1327-1336 (2006).

27. Taylor, S. N., Basile, L. A., Ebeling, M. \& Wagner, C. L. Intestinal permeability in preterm infants by feeding type: mother's milk versus formula. Breastfeed. Med. 4, 11-15 (2009).

28. Moore, S. A. et al. Intestinal barrier dysfunction in human necrotizing enterocolitis. J. Pediatr. Surg. 51, 1907-1913 (2016).

29. Usami, M. et al. Effect of eicosapentaenoic acid (EPA) on tight junction permeability in intestinal monolayer cells. Clin. Nutr. 20, 351-359 (2001).

30. Willemsen, L. E. M. et al. Polyunsaturated fatty acids support epithelial barrier integrity and reduce IL-4 mediated permeability in vitro. Eur. J. Nutr. 47, 183-191 (2008). 
Effect of polyunsaturated fatty acids on postnatal ileum development. . $P$ Singh et al.

31. Beguin, P., Errachid, A., Larondelle, Y. \& Schneider, Y.-J. Effect of polyunsaturated fatty acids on tight junctions in a model of the human intestinal epithelium under normal and inflammatory conditions. Food Funct. 4, 923 (2013).

32. Pendyala, S., Walker, J. M. \& Holt, P. R. A high-fat diet is associated with endotoxemia that originates from the gut. Gastroenterology 142, 1100-1.e2 (2012).

33. Lippestad, M., Hodges, R. R., Utheim, T. P., Serhan, C. N. \& Dartt, D. A. Resolvin D1 increases mucin secretion in cultured rat conjunctival goblet cells via multiple signaling pathways. Invest. Ophthalmol. Vis. Sci. 58, 4530-4544 (2017)

34. Zhao, J. et al. Preferential generation of 15 -HETE-PE induced by IL-13 regulates goblet cell differentiation in human airway epithelial cells. Am. J. Respir. Cell Mol. Biol. 57, 692-701 (2017).
35. Phillips, T. E., Stenson, W. F. \& Neutra, M. R. Lipoxygenase metabolites of arachidonic acid do not induce mucus secretion from rabbit intestinal goblet cells in vitro. Prostaglandins Leukot. Essent. Fat. Acids 37, 51-55 (1989).

36. Najm, S. et al. Effects of a lipid emulsion containing fish oil on polyunsaturated fatty acid profiles, growth and morbidities in extremely premature infants: a randomized controlled trial. Clin. Nutr. ESPEN 20, 17-23 (2017).

37. $\mathrm{Yu}, \mathrm{H} . \mathrm{-N}$. et al. Effects of fish oil with a high content of $\mathrm{n}-3$ polyunsaturated fatty acids on mouse gut microbiota. Arch. Med. Res. 45, 195-202 (2014).

38. Ghosh, S. et al. Fish oil attenuates omega- 6 polyunsaturated fatty acid-induced dysbiosis and infectious colitis but impairs LPS dephosphorylation activity causing sepsis. PLoS ONE 8, e55468 (2013). 